\title{
RECONSTRUCCIÓN DE DEFECTOS MANDIBULARES CON INJERTO DE CRESTA ILÍACA. INVESTIGACIÓN RETROSPECTIVA DE 24 CASOS
}

\section{RECONSTRUCTION OF MANDIBULAR DEFECTS WITH CRESTA ILÍACA INJERTO RETROSPECTIVE RESEARCH OF 24 CASES}

\author{
Palacios-Vivar Diego $^{1 *}$, Miranda-Villasana José ${ }^{2}$, Viteri-Luzuriaga Arturo ${ }^{3}$, Calderón-Lumbreras Angélica 4 \\ ${ }^{1}$ Cirujano Maxilofacial, servicio de Cirugía Maxilofacial del Hospital Regional "General Ignacio Zaragoza", ISSSTE, \\ Ciudad de México. México \\ 2 Maestro en Educación, Jefe de servicio de Cirugía Maxilofacial del Hospital Regional "General Ignacio Zaragoza”, \\ ISSSTE, Ciudad de México. México \\ ${ }^{3}$ Cirujano Maxilofacial, servicio de Cirugía Maxilofacial del Hospital Regional "General Ignacio Zaragoza”, ISSSTE, \\ Ciudad de México. México \\ ${ }^{4}$ Médico Interno de Pregrado, FacMed, Universidad Nacional Autonoma de México. México \\ *diegoepalaciosvivar@yahoo.com
}

\begin{abstract}
Resumen
INTRODUCCIÓN: Establecer el éxito, y conocer las razones del fracaso son pasos fundamentales para construir una medicina basada en evidencia que se pueda mejorar y replicar en otras manos en diferentes latitudes. Hecho por el cual presentamos un estudio sobre el manejo de los defectos mandibulares mediante injertos óseos. OBJETIVO: Evaluar los resultados del tratamiento de reconstrucción mandibular, mediante la utilización de injerto de cresta iliaca. MATERIALES Y MÉTODOS: La investigación se realizó en el Servicio de Cirugía Maxilofacial del Hospital Regional "Gral. I. Zaragoza" en el oriente de la Ciudad de México, de enero del 2002 a diciembre del 2012, con un seguimiento mínimo de 2 años de cada paciente. El grupo de muestra fue de 24 pacientes con diferentes etiologías que ocasionaron defecto óseo mandibular que requiera una reconstrucción mandibular con injerto de cresta iliaca, efectuadas por el mismo Cirujano Maxilofacial. RESULTADOS: Los pacientes mostraron un éxito del $87 \%$ en cuanto a estabilidad, integración y reabsorción ósea moderada. La infección posquirúrgica mediata fue la complicación más frecuente $20.8 \%$, de las cuales dos pacientes terminaron con fracaso y pérdida del injerto óseo. CONCLUSIONES: El injerto de cresta iliaca en conjunto de material de reconstrucción es un método confiable para realizar la reconstrucción del defecto mandibular, con una recuperación de la función mandibular predecible, y una posibilidad de rehabilitación.
\end{abstract}

Palabras clave: Defecto mandibular, injerto óseo autólogo, resección en bloque, reconstrucción mandibular.

\begin{abstract}
INTRODUCTION: Establish rate of success, understand the reasons of failure both fundamental steps to build an evidencebased medicine that may improve and be replicate in other hands at different latitudes. We present a study on the management of mandibular defects using iliac crest bone graft. OBJETIVE: Evaluate the results of treatment of mandibular reconstruction using iliac crest bone graft. MATERIALS AND METHODS: The research was conducted in the Maxillofacial Surgery Regional Hospital "Gral. I. Zaragoza" in eastern Mexico City formjJanuary 2002 to december 2012 with a minimum followup of 2 years each patient. The sample group was 24 patients with different etiologies caused mandibular bone defect requiring a mandibular reconstruction with iliac crest graft, performed by the same surgeon Maxillofacial. RESULTS: Patients showed a success rate of $87 \%$ in terms of stability, integration and moderate bone resorption. Mediate postsurgical infection was the most common complication $20.8 \%$, of which two patients ended with failure and loss of bone graft. CONCLUSIONS: The iliac crest graft is reliable method for the reconstruction of mandibular defect with a predictable recovery of mandibular function, and a possibility of oral rehabilitation.
\end{abstract}

Key words: Mandibular defect, autologous bone graft, block resection, mandibular reconstruction. 


\section{INTRODUCCIÓN}

La mandíbula es un hueso impar y flotante de la cara con forma de herradura, anatómicamente dividida en cuerpo, ángulo, rama, apófisis condilar y coronoides (Figura I). Contribuye al contorno facial, al definir el tercio inferior. Participa directamente en la oclusión, masticación, porción anterior de la caja de resonancia para el lenguaje articulado y vía aérea. ${ }^{1}$

Un daño mayor a la estructura mandibular puede ser causado por, accidentes automovilísticos, patología, proyectil de arma de fuego, osteomielitis. La reconstrucción mandibular es por definición la restauración del hueso mandibular, su dentición y el tejido blando que lo circunscribe. ${ }^{2}$ Este se puede alcanzar mediante una amplia variedad de procedimientos que dependen de la etiología, ubicación, la extensión de la deformidad, tipo de material de reconstrucción disponible y experiencia del equipo quirúrgico.,

El injerto óseo autólogo, es el tratamiento principal para reconstrucción mandibular. Si el defecto es menor puede tomarse de un sitio intraoral, y si es mayor un sitio extraoral distante. Los sitio a elección para toma del injerto autólogo son: costilla, calota, tibia, peroné, escapula, radio, y cresta iliaca posterior y anterior, siendo esta ultima la que consideramos como la ideal por su técnica, baja morbilidad, y la disponibilidad de hasta $50 \mathrm{cc}$ de hueso. ${ }^{4,5} \mathrm{Su}$ optima reconstrucción influye directamente en varias funciones diarias como: fonación, masticación, deglución lo cual repercute en la calidad de vida. ${ }^{6}$

Esta investigación se basa en los resultados obtenidos en la realización de 24 reconstrucciones mandibulares, con injerto de cresta iliaca estabilizado con placas y tornillos de reconstrucción de titanio. Tratados en el Servicio de Cirugía Maxilofacial del Hospital Regional "Gral. I. Zaragoza" del ISSSTE en el oriente de la Ciudad de México D.F., de enero del 2002 a diciembre del 2012, con un seguimiento mínimo

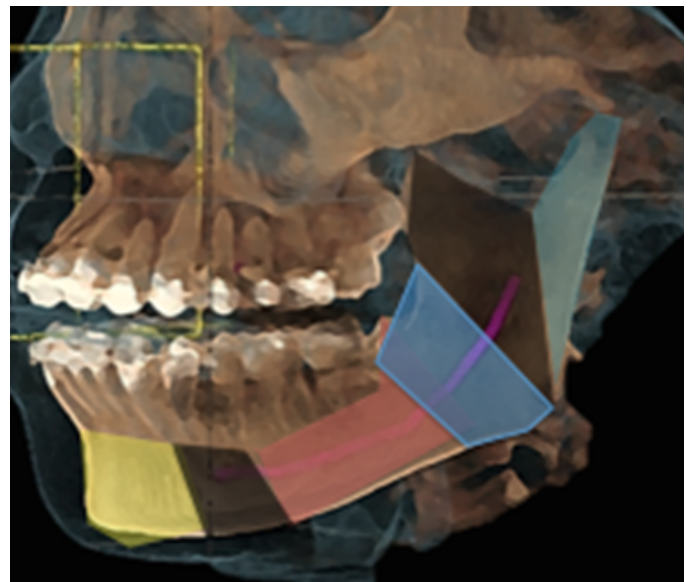

Fig. 1. División anatómica mandibular.

\section{Cuadro Clasificación defectos}

\begin{tabular}{ll}
\hline Alveolares & $\begin{array}{l}\text { Perdida de continuidad alveolar sin } \\
\text { perdida mandibular }\end{array}$ \\
Anterior & Segmentos de sínfisis, entre cúspides \\
& de caninos \\
Lateral & Segmentos desde la cúspide canina \\
& hasta la región retromolar \\
Posterior & Defecto del ángulo y rama mandibular \\
& con o sin alteración condilar.
\end{tabular}

Tabla 1. Clasificación de defectos óseos mandibular por zonas

de dos años en cada paciente.

\section{OBJETIVOS}

- Restablecer continuidad mandibular

- Restablecer una base ósea alveolar

- Corrección de los defectos de tejido blando.

- Permitir la rehabilitación oclusión

- Restablecer fisionomía mandibular

\subsection{TÉCNICAS DE RECONSTRUCCIÓN MANDIBULAR}

Se clasifican en cuatro categorías.

\section{Hueso autógeno}

o Injerto de hueso avascular

o Injerto de hueso pediculado

o Injerto osteomusculocutaneo vascularizados.

o Huesos prefabricado o prelaminado

\section{Distracción Osteogénica \\ 3. Materiales alopáticos \\ 4. Transporte óseo}

\subsection{GENERALIDADES PARA INJERTOS DE CRESTA ILIACA ANTERIOR}

El hueso autógeno, obtenido de la cresta iliaca anterior, posee propiedades osteoconductivas y osteoinductivas, necesita para su supervivencia un sitio receptor de tejido blando vascularizado y un tejido que provea de un cierre y los aislé del medio. De este sitio se puede obtener un bloque de $4 \mathrm{x}$ $5 \mathrm{~cm}$ o un volumen máximo de $50 \mathrm{cc}$. Su técnica es simple, contiene gran cantidad de hueso esponjoso. Presenta como desventaja principal el riesgo de parestesia del muslo lateral y una dificultad para deambular que desaparece después de las dos semanas. ${ }^{7,8}$ 


\section{MATERIALES Y MÉTODOS}

Esta investigación clínica de corte retrospectivo y longitudinal, se procedió a desarrollar la técnica de investigación documental y de campo en un grupo de veinticuatro pacientes con defectos mandibulares (Tabla I)los cuales fueron reconstruidos con injerto óseo de cresta iliaca, en el servicio de Cirugía Maxilofacial del 2002-2012.

Criterios de inclusión:

- Patología que involucre afectación ósea mandibular

- Fracturas mandibular con pérdida de segmento óseo

- Osteomielitis crónica severa con perdida ósea mandibular

Criterios de exclusión:

- Pacientes que vayan a someterse a radioterapia

- Pacientes con enfermedad sistémica de metabolismo óseo descompensada.

- Pacientes con tratamientos previos de injerto que fracasaron.

Criterios de eliminación:

- No cumplir con el seguimiento mínimo a dos años.

Se utilizó el protocolo de tratamiento para reconstrucción mandibular con injerto de cresta iliaca y fijación con placas de reconstrucción y tornillos de titanio en 24 pacientes que cumplieron los criterios seleccionados para el estudio. Previo de la intervención a todos los pacientes se les realizó tomografía computarizada, radiografía anteroposterior de cadera y métodos de estabilización de la oclusión, en seis de ellos una esteriolitografía mandibular.

Todas las cirugías se efectuaron bajo anestesia general e intubación orotraqueal con dos equipos de cirujanos maxilofaciales, uno para la resección mandibular y el segundo para la toma del injerto de cresta iliaca anterior. El abordaje y el material de reconstrucción mandibular se determino dependiendo de la dimensión del defecto a restaurar, en 19 procedimientos el abordaje mandibular fue extraoral, y 5 intraoral. De los cuales 22 se realizaron la reconstrucción primaria inmediata; dos pacientes se le realizaron en un segundo tiempo quirúrgico, uno de los cuales se utilizó un colgajo miocutaneo pectoral mayor bilateral.

Se ejecutó un control posquirúrgico tomando en cuenta: infección, reabsorción, rechazo, disminución de la radio densidad, utilizando estudio radiográfico con ortopantomografo tipo cone beam, al mes, tres y seis meses al año y a los dos años, clasificando el estadio del injerto en; cambios no apreciables radiográficamente, o cambios leves, moderados y severos.

\section{RESULTADOS}

La muestra fue de 24 pacientes, $15(62.5 \%)$ fueron del género masculino y $9(37.5 \%)$ género femenino. Los factores etiológicos fueron $10(41.6 \%)$ amelobastomas, 6 (16.6\%) queratoquistes, $3(12.5 \%)$ carcinoma de células escamosas

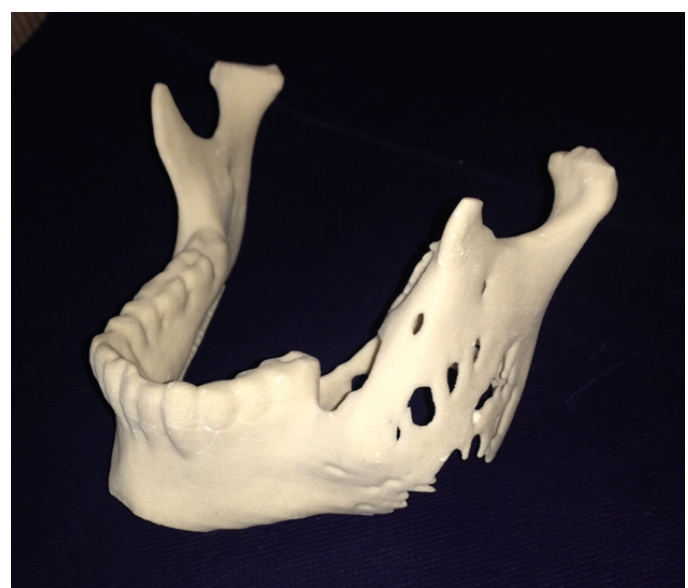

Fig. 2. Modelo estereolito mandibular de un carcinoma células escamosas.

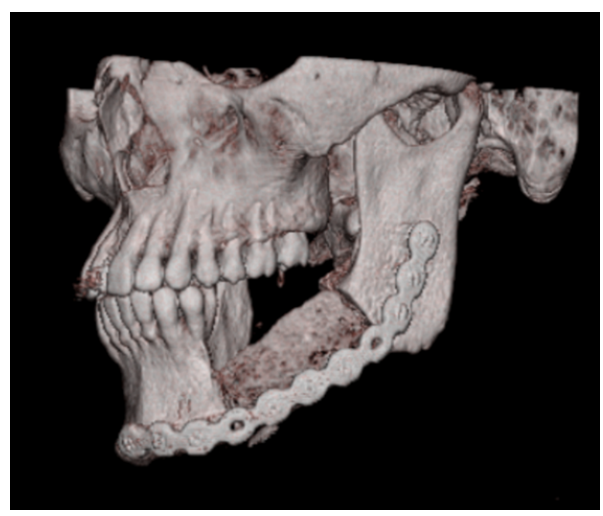

Fig. 3. Reconstrucción de tomografía en tercera dimensión: control injerto con placa de reconstrucción.

(Figura 2), 1 (4.16\%) mixoma, 4 (16.6) secuelas de fractura mandibular.

Todos los pacientes se realizaron la reconstrucción con injerto de cresta iliaca anterior, Morbilidad: A nivel de la zona donadora, un paciente se presento hematoma, fue drenado y evolución favorablemente, sin presencia de parestesia, paresia o infecciones. En cuanto a las zonas mandibulares afectadas (Gráfico I), se clasificaron por regiones mandibulares, encontrando la siguiente distribución: solo sínfisis uno paciente (4.16\%), sínfisis y parasínfisis dos pacientes $(8.3 \%)$, cuerpo siete pacientes $(29.1 \%)$, cuerpo y rama seis pacientes $(25 \%)$, parasínfisis y cuerpo tres pacientes $(12.5 \%)$, parasínfisis cuerpo y rama un paciente $(4.16 \%)$, cuerpo, rama y cóndilo dos pacientes $(8.3 \%)$. Se utilizó dos tipos de osteosíntesis: miniplacas de alto perfil en $4(17 \%)$ pacientes, y placas de reconstrucción en $20(83 \%)$ pacientes (Figura 3 y gráfico II).

Complicaciones: Se evaluó la presencia de infección, con revisiones clínicas periódicas. Cinco $(20.8 \%)$ pacientes presentaron procesos infecciosos, dos $(8.3 \%)$ en las primeras 


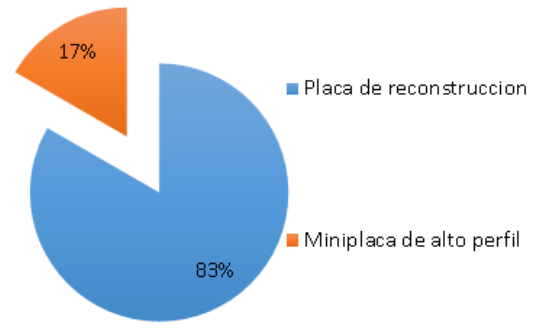

Graf. 1. Distribución por frecuencia del material de reconstrucción

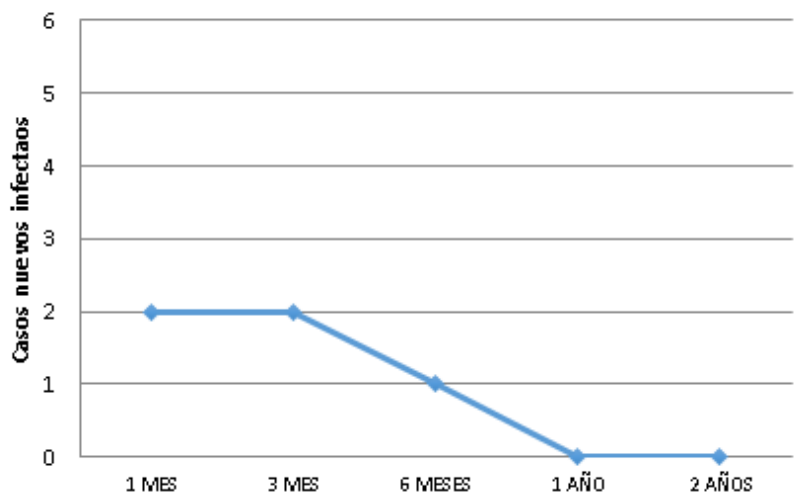

Graf. 2. Casos con infección en un periodo de dos años

dos semanas, dos $(8.3 \%)$ de manera mediata a las 6 semanas, uno $(4.16 \%)$ de manera tardía por rechazo un tornillo mal adaptado (Gráfico II). De estos pacientes, dos perdieron el injerto y se procedió su retiro. En el resto se lo conservó, luego de un reingreso hospitalario, se inicia con lavados con solución triple esquema, se progresa a clorexindina al $0.12 \%$ y antibioticoterapia parenteral. Se tomaron controles radiográficos en donde se evaluó el nivel de reabsorción en sus controles subsecuentes. En cuanto a la reabsorción al mes no hubo cambios en 21 ( $87.5 \%$ ) pacientes; tres (12.5 de ellos presentan leve disminución. Sin embargo a los tres meses sin cambios en $15(62.5 \%)$ casos; un cambio leve en seis (25\%) casos, moderado en dos $(8.3 \%)$, cambio severo en un $(4.16 \%)$ caso. A los seis $(25 \%)$ meses de control sin cambios en siete $(29.2 \%)$ pacientes; leve en $11(45.8 \%)$, moderado en dos $(8.3 \%)$, y severo en un $(4.16 \%)$ paciente. En un año se presentaron sin cambios en cuatro (17\%) pacientes; leve en $11(46 \%)$, moderado en seis $(25 \%)$, severo en tres (13\%) pacientes. A dos años del procedimiento los injertos presentaron los siguientes cambios: reabsorción leve en 13 $(54 \%)$, moderado en $8(33 \%)$, severo en $3(13 \%)$ pacientes (Gráfico III)

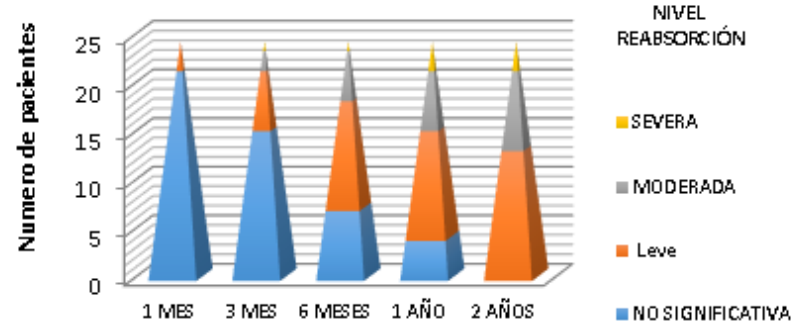

Graf. 3. Reabsorción del injerto mediante al cabo de 2 años.

\section{DISCUSIÓN}

La reconstrucción de los defectos mandibulares posee retos específicos de acuerdo a la etología que lo afecta y su extensión. Es un problema estructural, estético y funcional que amerita una adecuada planificación y contar de un equipo multidisciplinario. La indicación más común fue la reconstrucción secundaria a la resección de neoplasias benignas de grandes dimensiones (queratoquiste, ameloblastoma), seguido de neoplasias malignas y secuelas de fracturas complejas. ${ }^{5,9}$

En nuestro estudio se estableció un de éxito del $87 \%$ y un fracaso de tan solo el $13 \%$. Shaheen Anjum et al, reportan 15 casos de hemimandibulectomia, en los cuales se reconstruyo con injerto de cresta iliaca y costilla, presentando un éxito del $100 \% .{ }^{10}$ Malik reportó un éxito $90 \%$, y fracaso del $10 \%$, con un grupo de 30 pacientes. ${ }^{5}$ Szpindor, reportaron un éxito de $84 \%$, y fracaso del $16 \%$, con un grupo de estudio de 64 pacientes. ${ }^{11}$ Otro estudio de Zubing Li, con un grupo más extenso 242 pacientes, reportaron un éxito del $95.8 \%$, y un fracaso del $4.2 \%{ }^{9}$

El fracaso de nuestro estudio es representado por tres casos. Primer caso: masculino de 52 años de edad, con diagnóstico de carcinoma células escamosas, presento recidiva del tumor generando exposición del injerto. Segundo caso: masculino de 25 años, que presento dehiscencia de la herida y exposición del injerto, ocasionando su pérdida. Tercero: masculino de 41 años, con diabetes tipo II descontrolada, presento necrosis de los bordes de la herida, dehiscencia de manera temprana e infecciones recurrentes.

Existe una opinión divida, en el uso de injerto vascularido y no vascularizados para la reconstrucción mandibular. Autores como Marx refiere que el uso de un colgajo vascularizado no representa en realidad un avance en la reconstrucción ${ }^{12}$ y presenta un inconveniente importante que es la trombosis venosa, el factor causante de su fracaso. El uso de injerto no vascularizado provee de una mayor cantidad de hueso, genera continuidad ósea, adecuada altura alveolar, y la capacidad de ser soportar un implante endooseo. El éxito de los injertos según la literatura mundial 
va de un $38-80 \%$, con gran margen de acuerdo articulo 10 publicado. ${ }^{3,5,12}$ Un estudio realizado por Pogrel entre los 39 injertos vascularizados y 29 no vascularizados, el éxito de los vascularizado fue de un $95 \%$, en comparación con el de $76 \% 1$ de no vascularizado, un resultado importante se estableció que el rechazo fue mayor en aquellos en los que el defecto superior $\operatorname{los} 6 \mathrm{~cm}$. En cuanto a las desventajas, frente a los 12 injerto vascularizados, es la reabsorción lenta progresiva.

\section{CONCLUSIÓN}

El injerto de cresta iliaca anterior es la principal fuente de tejido óseo para reconstrucción mandibular, produce resultados predecibles, y con un alto porcentaje de éxito en defectos menores de $5 \mathrm{~cm}$. El seguimiento clínico-radiográfico es obligatorio a mediano y largo plazo. En casos de defectos en zonas dentadas valorar la posibilidad de rehabilitación mediante implantes.

Conflicto de intereses y financiamiento Los autores declaran no tener conflicto de intereses, haber cumplido con los requisitos de autoría y haber autofinanciado este artículo.

\section{Referencias}

1 Boyd J, Gullane P. Classification of mandibular defects. Plas Reconstruction Surg 1993; 92: 1266-1275.

2 Gendern E, Rinaldo A, Suarez C. Complications of free flap transfers for head and neck reconstruction following cancer resection. Oral Oncol 2004; 40: 979-984.

3 Goh B, Lee S, Tiderman H, Soelinga P, Mandibular reconstruction in adults: a review. Int J Oral Maxillofac Surg 2008; 37: 597-605.

4 Koch W, Yoo G, Goodstein M, Eisele D, Richtsmeier W, Price J. Advantages of mandibular reconstruction with the titanium hollow screw osseo-integrating reconstruction plate (THORP). Laringoscope 1994; 104: 545-552.

5 Malik A, Hassan S, Reconstruction of mandibular defects with autogenous bone grafts: A review of 30 cases. J. Ayub Med Coll Abbottabad 2011; 23(3): 82-85.

6 Zavalishina L, Karra N, Suliman W, El-Hakim M. Quality of life assesement in patients after mandibular resection and free fibula flap reconstruction. J Oral Maxillofac Surg 2014; 76: 1616-1626.

7 Myong H, Kim Y, Heo M, Lee S, Choi S, Kim M. Comparative radiologic study of bone density and cortical thickness of donor bone used in mandibular reconstruction. Oral Sur Oral Med Oral Pathol Oral Radiol Endod 2001; 91 (1): 2329.

8 Rana M, Warraich R, Kokemuller H, Reconstruction of mandibular defects-clinical retrospective research over a 10year period. Head Neck Oncol. 2011; 3 23(3): 1-7 .

9 Zubing L, Yifang Z, Sheng Y, Jibong Z, Shibin Y, Wnferng $Z$. Immediate reconstruction of mandibular defects: A retrospective report of 242 Cases. American Association of Oral and Maxillofacial Surgeon 2006; 06: 283-890.
0 Shaheen A. Treatment of reconstruction with iliac bone graft and rib graft in patients with mandibular defects. Pakistan Oral and Dental Journal 2013; 33 (3): 719-724.

1 Szpindor E. Evaluation of the usefulness of autogenous bone grafts in reconstruction of the mandible. Ann Acad Med Stein 1995; 41: 155-169.

2 Marks D, Hollinger J. Mandibular reconstruction. J Oral Maxillofac Surg 1993; 51: 466-479.
Recibido: 10 de Julio de 2016

Aceptado: 15 de Julio de 2016 
